\title{
Les aléas d'une activité transnationale. Les représentants du CNPF-MEDEF à Bruxelles et les marges de l'Eurocratie depuis 1973
}

The Uncertainty of Transnational Activities. The Representatives of French Employers in Brussels and the Sidelines of Eurocracy since 1973

\section{Yohann Morival}

\section{OpenEdition Journals}

Édition électronique

URL : http://journals.openedition.org/conflits/19639

DOI : 10.4000/conflits. 19639

ISSN : $1777-5345$

Éditeur :

CCLS - Centre d'études sur les conflits lilberté et sécurité, L'Harmattan

Édition imprimée

Date de publication : 31 décembre 2017

Pagination : $59-76$

ISBN : 978-2-343-14601-0

ISSN : $1157-996 X$

Référence électronique

Yohann Morival, « Les aléas d'une activité transnationale. Les représentants du CNPF-MEDEF à Bruxelles et les marges de l'Eurocratie depuis $1973 »$, Cultures \& Conflits [En ligne], 108 | hiver 2017, mis en ligne le 31 décembre 2019, consulté le 30 mars 2021. URL : http://journals.openedition.org/ conflits/19639; DOI : https://doi.org/10.4000/conflits.19639 


\section{Les aléas d'une activité transnationale. Les représentants du CNPF-MEDEF à Bruxelles et les marges de l'Eurocratie depuis 1973}

\section{Yohann MORIVAL 1}

Yohann Morival est maître de conférences en science politique (Université de Lille - CERAPS). Sa thèse, réalisée au Centre Maurice Halbwachs (ENS-EHESS), est intitulée "Les Europes du Patronat. L'enjeu "Europe" au sein des organisations patronales françaises depuis 1948 ". Il a notamment publié : "L'Europe dérange l'organigramme". Le travail de l'Europe au sein des organisations patronales françaises depuis 1956 ", Politique européenne, vol. 3, n 57, 2017 ; "La fabrique des légitimités européennes ", Critique internationale, $n^{\circ}$ 1, 2017 ; "Passage à Bruxelles et structuration nationale de l'intérêt européen au sein du CNPF ", Relations internationales, vol. 2, n 157, 2014.

T a confédération patronale française, qui a d'abord porté le nom de Conseil ـnational du patronat français (CNPF), puis celui de Mouvement des entreprises de France (MEDEF), dispose depuis 1973 d'un Bureau de représentation auprès de l'Union européenne (UE) situé à Bruxelles. Lorsqu'ils décrivent leur activité, ces délégués permanents insistent sur leur position aux marges de l'espace politique européen. Ils affirment ainsi : «je peux faire illusion pendant cinq minutes [lors d'un rendez-vous avec un représentant des institutions européennes], mais après je vais être largué 2 », "je suis persuadé qu'on m'a recruté parce que je ne connaissais rien à l'Europe ${ }^{3}$ ", ou encore, de manière contre-intuitive pour un représentant à Bruxelles, "si on reste dans le monde bruxellois, on est fichu ${ }^{4}$ ». Si la mobilité transnationale et le dévelop-

1. Je remercie vivement Laurent Jeanpierre et Laurent Bonelli pour leurs retours lors du congrès de l'AFSP ainsi que les deux évaluateurs anonymes de Cultures $E$ Conflits pour leurs commentaires sur une première version de ce texte.

2. Entretien avec un ancien délégué permanent à Bruxelles entre 1979-1988 et 1996-2003, mai 2010.

3. Entretien avec un ancien délégué permanent du CNPF à Bruxelles (1988-1996), mars 2012.

4. Entretien avec l'actuel directeur des affaires européennes du MEDEF, juin 2015. 
pement d'une activité multi-située ne sont pas également répartis selon les groupes sociaux ${ }^{5}$, il est peu fréquent que les difficultés soient éprouvées par les salariés d'une organisation patronale. En effet, plusieurs ressources permettant d'évoluer au sein de l'espace politique européen sont souvent associées à ces acteurs : expertise technique rare, connaissance fine du jeu institutionnel européen et capital social européen ${ }^{6}$. En contrepoint, les délégués permanents de la confédération patronale française à Bruxelles sont davantage caractérisés par le maintien d'une altérité nationale au sein d'un espace transnational. L'entrée par cette position européenne relativement peu européanisée invite à interroger plus largement les contraintes de l'action transnationale.

Les dirigeants du CNPF-MEDEF disposent de plusieurs moyens d'action en direction des institutions européennes, que ce soit par le biais de la confédération patronale européenne, l'UNICE, devenue BusinessEurope en 2007, ou par l'envoi régulier à Bruxelles d'experts du siège parisien ${ }^{7}$. Différents travaux récents ont ainsi documenté l'imbrication des sphères publique et privée dans le fonctionnement de l'Union européenne ${ }^{8}$ et au-delà 9 . Les délégués permanents à Bruxelles fournissent un éclairage nouveau sur des acteurs patronaux qui, tout en évoluant à Bruxelles, ne sont pas au cœur de ces processus d'influence. En comparaison avec les délégués permanents à Bruxelles d'autres confédérations nationales, ceux du CNPF-MEDEF disposent de moyens humains et financiers limités. Leur activité au sein du Bureau de représentation ne leur permet d'ailleurs pas de poursuivre une carrière à l'échelle européenne. Ils se maintiennent à la marge des métiers de l'Europe politique ${ }^{10}$. Ils donnent ainsi à voir les misères de position de l'Eurocratie, misères « relatives au point de vue de celui qui les éprouve en s'enfermant dans les limites du microcosme 11 », qui ont pu être documentées récemment pour les officiers militaires français 12 ou les assistants parlementaires ${ }^{13}$.

5. Wagner A-C., Les classes sociales dans la mondialisation, Paris, La Découverte, 2007.

6. Courty G. et H. Michel, «Groupes d'intérêt et lobbyistes dans l'espace politique européen : des permanents de l'Eurocratie ", in Georgakakis D. (dir.), Le champ de l'Eurocratie : une sociologie politique du personnel de l'UE, Paris, Économica, 2012, pp. 213-239.

7. Morival Y., Les Europes du Patronat Français. L'enjeu "Europe " dans les organisations patronales françaises depuis 1948, Thèse de doctorat, EHESS, 2015.

8. Laurens S., Les courtiers du capitalisme : milieux d'affaires et Bureaucrates à Bruxelles, Marseille, Agone, 2015.

9. France P. et A. Vauchez, Sphère publique, intérêts privés : enquête sur un grand brouillage, Paris, Presses de Sciences Po, 2017.

10. Georgakakis D. (dir.), Les métiers de l'Europe politique : acteurs et professionnalisations de l'Union européenne, Strasbourg, Presses universitaires de Strasbourg, 2002.

11. Bourdieu P., «L'espace des points de vue », in Bourdieu P. (dir.), La misère du monde, Paris, Seuil, 1993, pp. 9-11.

12. Boncourt T., "French military careers and European security integration: How internationalisation changes military socialisation ", Cooperation and Conflict, 2017, vol. 52, $\mathrm{n}^{\circ} 2$, pp. 241-260.

13. Michon S., Les équipes parlementaires des eurodéputés : entreprises politiques et rites d'institution, Luxembourg, Promoculture Larcier, 2014. 
L'entrée par la difficulté à légitimer une position transnationale au sein d'un groupe national vient compléter l'importante littérature sur le passage à Bruxelles. Plusieurs critères devant favoriser ou non le développement d'une action transnationale par un groupe national ont ainsi été identifiés dans cette littérature : la nature du cadre institutionnel dans lequel évolue le groupe d'intérêt national ; les réglementations politiques structurant son activité ou encore l'origine de ses moyens financiers ${ }^{14}$. L'opposition entre des «multilevel players ", acteurs collectifs capables d'agir à différentes échelles, et les acteurs agissant uniquement au niveau national ${ }^{15}$, se fait au détriment d'une attention portée aux difficultés propres à l'action multi-située. Or, le cas du Bureau du CNPF-MEDEF signale qu'au sein d'organisations où se développe une action multi-située, certains acteurs pourtant décrits comme transnationaux ne parviennent pas à accumuler des ressources spécifiques à cet espace et développent une activité qui ne s'y inscrit que partiellement.

Comprendre ces contraintes nécessite de multiplier les échelles d'analyse. Les délégués permanents à Bruxelles sont replacés au sein des concurrences internes à un espace européen de représentation des intérêts déjà structuré quand le bureau est mis en place. Néanmoins, une focale sur la seule échelle européenne aurait pour effet d'accentuer l'autonomie de l'espace bruxellois de la représentation des intérêts. L'action transnationale paraît difficilement dissociable du cadre de l'organisation nationale dans laquelle elle est produite. Les contraintes d'une activité transnationale proviennent également du fonctionnement de l'organisation et du sens donné à cette activité. De plus, l'action européenne éclaire les concurrences avec d'autres groupes nationaux. Dès lors, les délégués permanents à Bruxelles sont replacés à la fois au sein des concurrences internes aux espaces européen et français de représentation des intérêts patronaux et dans la division du travail de l'Europe interne au CNPFMEDEF.

Ce faisant, il est possible de rendre compte des multiples usages d'une présence européenne. Si la prédominance du prisme du lobbying pour appréhender l'action des groupes d'intérêt à l'échelle européenne ${ }^{16}$ passe sous silence la

14. Bennett R. J., «Business Routes of Influence in Brussels: Exploring the Choice of Direct Representation ", Political Studies, 1999, vol. 47, n² 2, pp. 240-257 ; Bernhagen P. et N.J. Mitchell, «The Determinants of Direct Corporate Lobbying in the European Union ", European Union Politics, 2009, vol. 10, n² 2, pp. 155-176; Kerremans B et J. Beyers, "Critical Resource Dependencies and the Europeanization of Domestic Interest Groups ", Journal of European Public Policy, 2007, vol. 14, n 3, pp. 909-920; Eising R., « Multilevel Governance and Business Interests in the European Union ", Governance, 2004, vol. 17, n 2, pp. 211245 ; Klüver H., « Europeanization of Lobbying Activities: When National Interest Groups Spill Over to the European Level ", Journal of European Integration, 2010, vol. 32, n 2, pp. 175-191.

15. Eising R., "Multilevel Governance and Business Interests in the European Union », op. cit.

16. Klüver H., Lobbying in the European Union: Interest Groups, Lobbying Coalitions, and Policy Change, Oxford, Oxford University Press, 2013 ; Kluger Rasmussen M., « The Battle for Influence: The Politics of Business Lobbying in the European Parliament », JCMS: Journal of Common Market Studies, 2015, vol. 53, n² 2, pp. 365-382 ; Richardson J., Mazey 
diversité des activités développées par ces groupes à l'échelle européenne ${ }^{17}$, il en est de même pour la présence de groupes nationaux à Bruxelles. Tenus à l'écart des activités de lobbying, les délégués permanents du CNPF-MEDEF s'investissent dans la production d'une parole destinée aux membres nationaux depuis Bruxelles. Ils participent ainsi à l'affirmation de la position de la confédération patronale française au sein de l'espace français de la représentation des intérêts patronaux. Les enjeux d'une présence européenne essentiellement dédiée à l'espace national depuis Bruxelles éclairent in fine le fonctionnement de la confédération patronale française.

Plusieurs types de matériaux empiriques sont mobilisés pour appuyer la démonstration. Des entretiens semi-directifs ont été menés avec l'ensemble des délégués permanents du CNPF-MEDEF à Bruxelles depuis 1973 ainsi qu'avec les différents responsables des affaires européennes basés à Paris. Ces acteurs ont été rencontrés à plusieurs reprises entre fin 2010 et jullet 2017. De plus, des documents d'archives, comme des budgets, des notes de service ou des procès-verbaux, ont été consultés dans les archives de la confédération patronale française afin de replacer la position de délégué permanent dans le cadre contraint de l'organisation pour lequel il agit. En complément d'approches qualitatives décrivant la structuration d'un espace bruxellois de la représentation des intérêts ${ }^{18}$, cette approche qualitative est la plus à même de rendre compte des tensions structurant l'activité transnationale en tenant ensemble les dynamiques nationales et européennes.

L'étude des frontières de l'action transnationale se fait en deux temps. Tout d'abord, nous analysons la structuration particulière du Bureau bruxellois de la confédération patronale française à distance des institutions européennes. Si cette position éclaire la diversité des activités menées, elle explique également la difficulté de ces représentants à accumuler des ressources européennes. Ensuite, l'étude des dynamiques de maintien de ce Bureau marginal éclaire la diversité des usages d'une présence à Bruxelles. Pour les dirigeants de la confédération patronale française, dont le lobbying passe par d'autres canaux, il s'agit notamment de répondre aux demandes de leurs membres et de certains membres du gouvernement.

S., "Shooting Where the Ducks Are: EU Lobbying and Institutionalised Promiscuity », in Richardson J. et Mazey S. (dir.), European Union: power and policy-making, Oxon/New York, Routledge, 2015, pp. 419-443.

17. Michel H., "Businesseurope au-delà du "lobbying" : le travail d'intégration européenne d'une organisation patronale ", Critique internationale, 2013, vol. 2, n 59, pp. 133-155; Milet M., La représentation des intérêts en Europe comme anti-lobbying ? L'UEAPME et la revendication des petites et moyennes entreprises, Habilitation à diriger des recherches, Université Paris 2, Paris, 2015.

18. Berkhout J. et D. Lowery, "The changing demography of the EU interest system since 1990 ", European Union Politics, 2010, vol. 11, n 3, pp. 447-461; Michel H. et S. Laurens, "Socio-histoire d'un espace de représentation européenne des intérêts patronaux (19602004) ", in Michel H. (dir.), Représenter le patronat européen : Formes d'organisation patronale et modes d'action européenne, Bruxelles, Peter Lang, 2013, pp. 23-45. 


\section{Les marges de l'action transnationale}

Si le «Bureau de représentation auprès des institutions européennes » est un type de structure répandu au sein des organisations non gouvernementales, des entreprises ou des groupes d'intérêt, il ne renvoie pas à un type de fonctionnement standardisé ou à un ensemble défini de pratiques. Certains Bureaux rassemblent des experts sectoriels et sont des espaces de production de prises de position européennes ${ }^{19}$. D'autres regroupent des acteurs européanisés devant porter une cause auprès des institutions européennes, comme l'adhésion de la Turquie à l'Union européenne ${ }^{20}$. Le Bureau de représentation du CNPF-MEDEF relève d'un autre modèle. Il fonctionne avec des salariés peu nombreux, jamais associés à la prise de décision et qui entretiennent de surcroît peu de relations avec les représentants des institutions européennes. Acteurs évoluant au sein de l'espace politique européen sans toutefois capitaliser sur cette position européenne, les délégués permanents du CNPFMEDEF éclairent la définition d'une position de représentant européen dont l'activité demeure en partie régie par des logiques nationales.

\section{Un Bureau à distance des institutions européennes}

Les régulières recompositions de l'activité du Bureau à Bruxelles du CNPF-MEDEF signalent la distance de ses délégués permanents avec les institutions européennes. Leur activité est davantage centrée sur les relations avec les fédérations nationales membres du CNPF-MEDEF que sur les liens avec les fonctionnaires européens.

Lorsqu'il est évoqué pour la première fois en 1958, le Bureau a pour ambition de constituer une structure délocalisée de prise en charge des sujets européens à Bruxelles par et pour le CNPF. Toutefois, le chargé des affaires européennes recruté refuse de s'installer à Bruxelles. Le projet d'un Bureau de représentation est alors mis en veille. Pendant près de dix ans, le chargé des affaires européennes du CNPF ou son adjoint, tous deux basés à Paris, font plusieurs fois par mois le trajet vers Bruxelles, principalement pour assister aux réunions des délégués permanents de l'UNICE. La constitution du premier Bureau du CNPF à Bruxelles en 1973 est justifiée par la nécessité de mieux contrôler l'activité des Britanniques qui rejoignent la Communauté économique européenne (CEE) en 1973 21. L'enjeu est de suivre l'action de

19. Berny N., "Le lobbying des ONG internationales d'environnement à Bruxelles ", Revue française de science politique, 2008, vol. $58, \mathrm{n}^{\circ} 1$, pp. 97-117.

20. Visier C., «Les représentants d'intérêt turcs à Bruxelles : la socialisation à travers les modalités d'articulations de différentes appartenances ", in Michel H. et C. Robert (dir.), La fabrique des "Européens». Processus de socialisation et construction européenne, Strasbourg, Presses universitaires de Strasbourg, 2010, pp. 77-98.

21. Pour plus de détails sur la genèse de ce Bureau : Morival Y., " Pourquoi et comment investir Bruxelles ? ", in Michel H. (dir.), Représenter le patronat européen. Formes d'organisation patronale et modes d'action européenne, op. cit., pp. 96-120. 
nouveaux membres avec lesquels les représentants du CNPF ont eu de nombreux désaccords.

La liaison avec l'UNICE et le suivi de l'activité des représentants britanniques se font avec un nombre limité de salariés. En 1979, le Bureau du CNPF se compose d'un représentant et d'une secrétaire. Au même moment, la délégation de la confédération patronale italienne à Bruxelles, la Confindustria, comptait entre quinze et vingt personnes. Le Bureau de représentation de la Confindustria a été ouvert dès la fin des années 1950 en raison notamment de l'impossibilité de réaliser des aller-retour rapides entre Bruxelles et Rome ${ }^{22}$. Ce Bureau constitue alors un «véritable service des affaires européennes » dont les membres sont associés à l'ensemble des décisions européennes de la confédération italienne et entretiennent des relations régulières avec les représentants des institutions communautaires ${ }^{23}$. De même, l'ensemble BDI-BDA, les deux organisations patronales allemandes, employait à cette période sept ou huit personnes pour leur Bureau de Bruxelles 24. Analyser l'activité du Bureau nécessite de se détacher d'une logique strictement comptable selon laquelle plus il y aurait de salariés, plus l'activité serait importante. Cette approche passe sous silence les différentes activités développées au sein du Bureau de représentation. Le Bureau de représentation de la Confindustria s'apparente à un véritable service des affaires européennes et celui des organisations allemandes représente une structure nouvelle regroupant deux organisations distinctes et n'étant donc pas soumis aux concurrences des services européens de chaque organisation. En revanche, le Bureau à Bruxelles du CNPF coexiste avec un service des affaires européennes situé au sein du siège parisien et ses membres s'investissent dans d'autres actions.

Au début des années 1980, une nouvelle activité est développée au sein du Bureau du CNPF à Bruxelles : le suivi de sujets à la demande de représentants de fédérations sectorielles membres. De nombreuses fédérations adhérèrent à ce projet : il y en avait dix-huit en 1991 et jusqu'à vingt-cinq à la fin des années 1990. Ces missions de veille étaient réalisées contre une cotisation supplémentaire permettant l'embauche de nouveaux salariés. Le Bureau du CNPF à Bruxelles s'étoffe pour compter au maximum une petite dizaine de salariés. Cette augmentation du nombre de salariés ne résulte pas d'un investissement direct plus important de la confédération patronale à Bruxelles. Effectuant des missions à la demande de fédérations membres, ces salariés ne participent pas directement à l'activité européenne du CNPF. La ligne « salariés directement

22. Outre la distance avec Bruxelles, l'investissement rapide de Bruxelles par les dirigeants patronaux italiens tient aussi à la volonté d'affirmer une expertise propre face à l'État italien : Petrini F., Il liberismo a una dimensione: la Confindustria e l'integrazione europea, 19471957, Milan, Franco Angeli, 2005, 333 p.

23. Archives regroupées par Yohann Morival, Patronats en Europe, rapport du CNPF, 1990.

24. Entretien avec un ancien délégué permanent à Bruxelles entre 1979-1988 et 1996-2003, avril 2014. 
rémunérés » du tableau 1 ci-dessous, qui résulte d'un travail de confrontation entre les données du CNPF et les documents d'archives ${ }^{25}$, donne une meilleure idée des moyens humains des différents Bureaux de représentation de confédérations patronales nationales en 1993. Avec deux salariés dont uniquement un cadre et une secrétaire, le CNPF dispose des moyens humains les plus restreints.

\begin{tabular}{ccccccc}
\hline Organisation & BDA & BDI & CEOE & Confindu. & CNPF & CBI \\
\hline Salariés & 3 & 6 & 7 & 12 & 8 & 9 \\
\hline $\begin{array}{c}\text { Salariés } \\
\text { directement } \\
\text { rémunérés }\end{array}$ & 3 & 6 & 7 & 6 & 2 & 5 \\
\hline
\end{tabular}

Tableau 1 : Nombre de permanents des Bureaux de représentation à Bruxelles en 1993

Le modèle du Bureau de représentation comme prestataire de service est remis en cause au début des années 2000 . À ce moment, plusieurs fédérations sectorielles européennes encouragent leurs membres nationaux à ouvrir des Bureaux à Bruxelles en propre et leur fournissent un soutien logistique. Peu à peu, le Bureau permanent du MEDEF perd l'ensemble des membres qui finançaient des salariés en son sein. Cette évolution explique le resserrement des effectifs pour la période actuelle, où le Bureau du MEDEF n'emploie plus que quatre personnes, dont deux cadres. Ces salariés répondent à des demandes ponctuelles du siège parisien, comme la recherche d'informations sur un point ou l'organisation de visites du Parlement européen pour les membres. Les différences entre les organisations se maintiennent dans le temps, comme le confirment les chiffres de 2010 recensés dans le tableau 2 cidessous, comprenant à la fois le nombre de cadres et le total des employés 26 . La baisse des effectifs du Bureau du MEDEF en comparaison avec les chiffres de 1993 est d'autant plus marquante que, dans le même temps, les représentations des autres patronats se sont renforcées, ou du moins maintenues.

25. Archives réunies par Yohann Morival, Patronats européens à Bruxelles, rapport du CNPF, 1993.

26. Archives réunies par Yohann Morival, MEDEF, « La voix des organisations professionnelles et des entreprises françaises à Bruxelles ", document de la Commission Europe du MEDEF, 2011. 


\begin{tabular}{ccccc}
\hline Organisation & BDI-BDA & CEOE & Confindu. & MEDEF \\
\hline Salariés & 14 & 10 & 12 & 4 \\
\hline Cadres & 10 & 6 & 7 & 2 \\
\hline
\end{tabular}

Tableau 2 : Nombre de permanents des Bureaux de représentation à Bruxelles en 2010

Dès lors, un Bureau de représentation d'une confédération patronale à Bruxelles n'est pas uniquement un espace où se définissent et se mettent en œuvre les différentes stratégies d'influence. Depuis 1973, l'activité du délégué permanent de la confédération patronale française à Bruxelles a été partagée entre trois missions principales : la liaison avec les activités de l'euro-groupe, l'UNICE puis BusinessEurope ; la réalisation de missions de veille ou de conseil pour les organisations membres de la confédération patronale ; et la réalisation de missions ponctuelles sur demande du siège parisien (recherche d'informations, réalisation d'un benchmarking entre plusieurs pays européens, préparation d'une visite, etc.). En tenant éloignés les délégués permanents des institutions européennes, ces activités ne leur permettent pas d'accumuler les ressources légitimes au sein de l'espace politique européen.

\section{Des représentants bruxellois peu européens}

Les acteurs évoluant au sein des différents métiers de l'Europe politique appartiennent souvent à une élite, tant du point de vue de la rémunération que du niveau de diplôme. La mobilité professionnelle entre ces métiers est fréquente. Ainsi, les représentants à Bruxelles des régions de différents pays membres, qui évoluent souvent dans des structures aux effectifs et aux moyens limités, quittent leur poste après trois à cinq ans pour une autre position, plus valorisée, au sein de l'Eurocratie ${ }^{27}$. Les délégués permanents du CNPFMEDEF, qui évoluent également au sein d'un Bureau de représentation avec des effectifs et des moyens limités, ne bénéficient pas de la même mobilité. Leur difficulté à acquérir une expertise européenne ou un capital social particulier éclaire les marges d'un éventuel « espace professionnel des affaires européennes 28 ».

27. Costa $\mathrm{O}$., «Les représentants des entités infra-étatiques auprès de l’Union. Processus de professionnalisation diversifiés et intérêts communs ", in Georgakakis D. (dir.), Les métiers de l'Europe politique : acteurs et professionnalisations de l'Union européenne, op. cit., p. 159.

28. Büttner S., Leopold L., Mau S. et M. Posvic, «Professionalization in EU Policy-Making? The topology of the transnational field of EU affairs ", European Societies, 2015, vol. 17, n 4, pp. 569-592. 
L'absence de carrière européenne des délégués permanents de la confédération patronale française étonne a priori tant ils disposent de ressources partagées par plusieurs acteurs de l'Eurocratie. Les délégués permanents du CNPF-MEDEF à Bruxelles sont diplômés de filières sélectives, comme les instituts d'études politiques (IEP) ou les écoles d'ingénieurs, et sont issus de la bourgeoisie économique. Néanmoins, aucun d'entre eux n'a suivi une formation dédiée aux enjeux européens. Le premier à occuper le poste, MichelCasati Olier, est un ancien ingénieur textile. Après avoir été chargé des relations avec les États-Unis au sein du CNPF, il est nommé à Bruxelles en 1973 sans connaissance sur le fonctionnement des institutions européennes. Son successeur, Bruno Vever, le remplace en 1979. Après avoir été diplômé de Sciences-Po Paris, où il n'avait pas suivi un cursus spécialisé sur les questions européennes, Bruno Vever intègre la direction des affaires sociales internationales du CNPF, où il ne suit cependant que partiellement les sujets européens, traitant uniquement de la dimension sociale. Robert Fries, qui lui succède en 1988, est diplômé de l'école de chimie de Paris. Il estime être arrivé à Bruxelles un peu par hasard et sans aucune connaissance du système européen. MarieChristine Vaccarezza, l'actuelle déléguée permanente, est diplômée en droit de l'environnement. Elle a dans un premier temps cherché à travailler en France, et ce n'est qu'à la fin d'un premier contrat au ministère de l'Environnement qu'elle s'est résolue à partir à Bruxelles, où elle a été journaliste avant de rejoindre le MEDEF.

Après leur passage à Bruxelles, ces délégués permanents ont repris une activité dans le cadre national où, dans la plupart des cas, les sujets européens n'occupaient pas une place centrale. Après plus de six ans à Bruxelles, Michel Casati-Ollier revient au siège parisien où il se charge des relations commerciales avec l'Australie. Robert Fries, licencié après huit ans à Bruxelles, ne trouve pas de nouvel emploi et passe quelques années au chômage avant de prendre sa retraite. Bruno Vever retrouve le siège parisien en tant que directeur des affaires européennes. Il est licencié quelques années après et reste ensuite au chômage. Tous les responsables du Bureau du CNPF débutent leur carrière dans le cadre national et aucun ne reste à Bruxelles après son passage par le Bureau permanent. L'action à l'échelle européenne ne va pas de pair avec l'accumulation des ressources propres à cet espace.

La distance de ces délégués avec les institutions communautaires est également une distance avec les ressources légitimes de l'espace politique européen. En effet, les agents de la Commission européenne ont joué un rôle important dans la constitution d'un espace européen de représentation des intérêts organisé autour des institutions européennes ${ }^{29}$. L'investissement auprès de ces agents permet notamment aux représentants patronaux d'acqué-

29. Michel H. et S. Laurens, «Socio-histoire d'un espace de représentation européenne des intérêts patronaux (1960-2004)», op. cit. 
rir un «capital bureaucratique européen », valorisé à la fois dans l'espace européen de la représentation des intérêts et au sein des groupes patronaux européens ${ }^{30}$. Dans les nombreuses relations entre représentants d'intérêts et membres des institutions européennes se joue, tout autant que l'influence, la production de l'européanité des acteurs ${ }^{31}$. Les délégués permanents du CNPFMEDEF témoignent du maintien d'acteurs faiblement européanisés dans cet espace professionnel transnational. Si Didier Georgakakis a signalé que des acteurs, nommés « intermittents de l'Eurocratie », parviennent à légitimer leur position au sein de l'Eurocratie en mobilisant principalement des ressources nationales, les délégués permanents de la confédération patronale française sont des acteurs nationaux qui, tout en maintenant une position transnationale, restent en marge du jeu institutionnel européen. Cette logique nationale de maintien au sein de l'espace européen de représentation des intérêts s'explique à la fois par l'inscription de leur activité dans l'espace national et par les limites que posent au développement de leur activité les concurrences internes à l'espace européen de la représentation des intérêts.

\section{L'espace concurrentiel de la représentation des intérêts patronaux à Bruxelles}

Lorsque le Bureau du CNPF à Bruxelles est fondé en 1973, les relations avec les représentants des institutions européennes pour la confédération patronale française sont déjà prises en charge par d'autres. Au sein du CNPF tout d'abord, où les permanents spécialisés sur des sujets techniques précis agissent à la fois aux échelles européenne et nationale. À l'échelle européenne ensuite où, à cette période, plusieurs permanents de la confédération patronale française se mobilisent depuis plusieurs années au sein de l'espace politique européen par le biais de la confédération patronale européenne, l'UNICE. L'investissement français est particulièrement marqué dans les premières années de la confédération patronale européenne, où plusieurs représentants du CNPF occupent des positions de pouvoir ${ }^{32}$. Les dirigeants du CNPF, privilégiant leur investissement au sein de la confédération patronale européenne, accordent d'ailleurs un budget limité au Bureau de représentation, environ cinq fois inférieur à la cotisation versée à l'UNICE. L'activité du Bureau de représentation se développe dans un espace européen déjà investi par d'autres représentants de la confédération patronale française. Ces autres formes de représentation des intérêts du CNPF à Bruxelles contraignent le développement du Bureau.

30. Laurens S., Les courtiers du capitalisme, op. cit.

31. Michel H., «La représentation du patronat européen à Businesseurope. Des présidents sans européanité », in Michel H. (dir.), Représenter le patronat européen : Formes d'organisation patronale et modes d'action européenne, op. cit., pp. 249-262.

32. Morival Y., «La fabrique des légitimités européennes : les acteurs de la confédération patronale européenne depuis $1952 »$, Critique internationale, 2017, vol. 1, n 74, pp. 33-51. 
Alors que les euro-groupes, comme l'UNICE-BusinessEurope, tentent de concilier la diversité des points de vue nationaux ou sectoriels pour garantir une prise de position européenne ${ }^{33}$, la délégation permanente du CNPFMEDEF devient un espace d'affirmation d'une prise de position nationale européenne. Dans les entretiens, l'actuelle représentante permanente du MEDEF précise son activité. Si le fond du dossier est traité au sein du siège parisien, elle doit trouver l'information :

«Par exemple, en fin de semaine débute le Conseil européen. Il y a sur la table un accord de libre-échange avec le Japon. Le MEDEF, comme le patronat italien, n'est pas favorable. (...) Je ne rentre pas sur le côté technique de l'accord de libre-échange, sur le fond c'est Paris qui se prononce, je donne juste l'information qu'a priori le président français et Monti [le président du Conseil italien] vont lâcher prise. Tout mon travail, c'est d'obtenir ces informations ».

Surtout, elle ajoute que le cœur de son activité se trouve dans sa capacité à décrypter l'information:

« Le texte, il fait huit pages en anglais, je ne vais pas l'envoyer comme ça. On est dans une situation où obtenir un document ce n'est pas le plus compliqué, mais ce qui fait partie du travail, et là où c'est important d'être basé à Bruxelles, c'est dans la sélection de l'information. Il faut pouvoir être reconnu comme un interlocuteur compétent. Il faut savoir décrypter l'information ${ }^{34}$ ».

Les délégués permanents disposent d'un sens pratique européen qui leur permet de comprendre l'information et de la contextualiser pour la rendre plus claire pour des experts parisiens qui n'évoluent que temporairement dans l'espace politique européen. De la même manière que les correspondants permanents de plusieurs médias auprès des institutions européennes sont confrontés au fait que «ce qui fait sens pour eux n'en a que très peu pour ceux à qui ils s'adressent ${ }^{35}$ », les délégués permanents de la confédération patronale doivent rendre les enjeux européens intelligibles à leurs interlocuteurs nationaux. Pour cela, ils doivent rester attentifs aux priorités de ces derniers. Les délégués permanents du CNPF-MEDEF appréhendent les questions européennes à partir d'une grille de signification nationale. On comprend alors mieux la formule a priori paradoxale d'un délégué permanent basé à Bruxelles : «Si on reste dans le monde bruxellois, on est fichu ${ }^{36}$ ».

33. Laurens S., "Make it E.U. friendly ", Sociétés contemporaines, 2013, $\mathrm{n}^{\circ} 89$, $\mathrm{n}^{\circ} 1$, pp. 17-46.

34. Entretien avec l'actuelle déléguée permanente du MEDEF à Bruxelles, octobre 2012

35. Baisnée O., « "L’Europe, ça ne s'improvise pas.” La socialisation professionnelle des correspondants auprès de l'Union européenne comme socialisation politique », in Michel $\mathrm{H}$. et $\mathrm{C}$. Robert (dir.), La fabrique des "Européens" : processus de socialisation et construction européenne, Strasbourg, Presses universitaires de Strasbourg, 2010, p. 375. 
Plus encore, il leur faut être au fait des dynamiques internes à la confédération patronale française. La sélection de l'information évolue en effet selon le type d'interlocuteur et sa position dans l'organisation:

«Il faut trouver l'information pertinente pour l'interlocuteur à Paris. Et c'est d'autant plus délicat qu'on couvre un scope très large à Paris, de la présidence aux chargés de mission. C’est très large. On va de la responsable protection des données, aux directeurs des affaires économiques, le délégué général voire la présidente. Selon l'interlocuteur, on reste dans du très technique qui n'appelle pas de commentaire de notre part, car il faut juste que je lui donne l'information qu'il n'a pas - comme ce que pensent les autres patronats, pourquoi tel patronat a changé d'avis, parce que sur le fond, il connait mieux le texte que moi - à du plus général qu'on commente pour la présidence par exemple 37 ».

L'apport du délégué permanent réside dans sa capacité à fournir une information européenne faisant écho aux priorités nationales et s'adaptant au fonctionnement du groupe composé de différents services et de différents niveaux d'acteurs (de l'expert technique à la présidente en passant par le président d'une commission intermédiaire).

La légitimité de ces délégués permanents réside dans cette capacité à choisir ce qui fait sens dans le groupe national. Ils concilient alors investissement transnational et ancrage national. La différence est marquée avec des syndicalistes français évoluant au sein de la Confédération européenne des syndicats qui non seulement adoptent un point de vue européen sur les pratiques nationales, regrettant ainsi les «divisions du syndicalisme français ", mais pour qui les ressources construites dans le cadre national sont relativement démonétisées ${ }^{38}$. Dès lors, analyser l'activité de ces délégués permanents du CNPFMEDEF implique de réinscrire leur activité non seulement dans les concurrences internes à l'espace européen de représentation des intérêts, mais également dans le fonctionnement du siège parisien de la confédération patronale française.

\section{Les logiques nationales de l'action européenne}

Les modalités de la division du travail sur les sujets européens au sein du CNPF-MEDEF contraignent la définition du rôle de délégué permanent à Bruxelles. En effet, malgré leur position à Bruxelles, ils restent en marge de la prise de décision sur les sujets européens au sein de la confédération patronale.

36. Entretien avec l'actuel directeur des affaires européennes du MEDEF, juin 2015.

37. Entretien avec l'actuelle déléguée permanente du MEDEF à Bruxelles, octobre 2012.

38. Wagner A-C., Vers une Europe syndicale : une enquête sur la Confédération européenne des syndicats, Bellecombe-en-Bauges, Edition du Croquant, 2005, p. 81. 
Plus que le constat d'une position marginale dans le travail de l'Europe à la fois aux échelles nationale et européenne, il importe d'interroger les logiques de son maintien. La position des délégués permanents à Bruxelles est légitimée par les attentes des membres nationaux du CNPF-MEDEF, mais aussi par des prises de position de membres du gouvernement français qui estiment que la confédération patronale française n'est pas assez active à Bruxelles. Le maintien d'une action transnationale essentiellement dédiée à l'espace national fournit des informations sur le fonctionnement de la confédération patronale française.

\section{Une position dominée dans la division du travail national}

Le délégué permanent est le représentant officiel de la confédération patronale française auprès de l'UNICE puis de BusinessEurope. Ce rôle s'accompagne d'une participation aux réunions bimensuelles des délégués permanents de l'ensemble des confédérations nationales membres de l'UNICEBusinessEurope. Le représentant à Bruxelles y suit les grandes lignes de l'activité, mais ne porte aucun sujet en propre. En effet, le Bureau bruxellois de la confédération patronale n'a jamais été conçu comme un espace semi-autonome d'activité européenne. La représentation de la confédération patronale française dans l'ensemble des réunions thématiques de l'UNICEBusinessEurope est assurée par des salariés du siège parisien spécialistes du sujet traité. Par exemple, pour un sujet concernant le droit de la concurrence, le MEDEF est représenté auprès de ses interlocuteurs institutionnels européens par un salarié spécialisé sur ce sujet et basé à Paris, et non par le délégué permanent à Bruxelles. Pour les relations avec le Parlement européen en revanche, il arrive que les membres du Bureau européen viennent en appui 39. Comme le résume le directeur des affaires européennes du MEDEF :

«Une structure comme le MEDEF, dans l'idéal, il faudrait qu'elle ait vingt personnes à Bruxelles : une pour l'environnement, une pour l'énergie, une pour la concurrence... Les Allemands ont une grosse structure parce que les gens sont à Bruxelles et pas à Berlin. Nous, les gens sont à Paris».

À la différence d'autres intermédiaires de l'international, notamment pour les professions juridiques ${ }^{40}$, les délégués permanents du CNPF-MEDEF ne parviennent pas à capitaliser sur leur position.

39. Morival Y., "Ceux qui font du Parlement européen une institution "politique" ", in Michon S. (dir.), Le Parlement européen au travail, Rennes, Presses universitaires de Rennes, à parâ̂tre.

40. Dezalay Y. et B. Garth, Global prescriptions: the production, exportation, and importation of a new legal orthodoxy, Ann Arbor, University of Michigan Press, 2002. 
Le délégué permanent agit davantage comme un entremetteur que comme un représentant, comme le précise le délégué permanent du CNPF à Bruxelles de 1979 à 1988 :

\begin{abstract}
«On traite de tout à Bruxelles, il faudrait être spécialiste de tout, ce qui n'est pas possible. Nous, on est capable de savoir qu'en ce moment, le gros sujet à Bruxelles c'est ça et que les personnes qui s'occupent de ça c'est untel et untel. Et après, je peux faire illusion en discutant avec elles pendant cinq minutes, mais au bout de dix minutes, je vais être largué... Par contre, si j’amène le spécialiste de Paris, même s'il n'a pas suivi directement cette affaire, il va comprendre ce que va dire l'autre parce qu'il est familier de ces problèmes ${ }^{41}$ ».
\end{abstract}

Le délégué permanent constitue un soutien pour l'action des experts thématiques du siège parisien. Une anecdote relatée par un ancien délégué permanent confirme leur faible implication dans la prise en charge des sujets européens :

\begin{abstract}
«Une fois, j’ai rencontré l'ancien dirigeant d'Axa, M. Bébéar, et il me dit: "Vous savez, je ne partage pas du tout la position du CNPF sur telle question", je ne sais plus quelle était la question. Je lui dis alors "bien, Monsieur le Président". Mais peut-être à ce moment-là, parce qu'il y avait une discussion, $j$ 'aurais dû prendre la parole pour défendre la position du CNPF, mais j'en étais incapable. Ça illustre assez ma position, j'en étais incapable, je n'étais pas payé pour ça, je n'avais pas reçu de formation pour ça et je n'avais pas les éléments. Ça situe assez bien quelle était ma position à l'époque 42 ».
\end{abstract}

Le monopole de la présence quotidienne à l'échelle européenne n'accorde pas au délégué permanent à Bruxelles un monopole de la parole sur ces sujets, ni même une expertise reconnue au sein de son organisation. Les salariés du Bureau européen ne sont pas associés à la définition des prises de position européennes de la confédération patronale, qui se fait exclusivement avec des acteurs du siège parisien.

L'entrée par les délégués permanents du CNPF-MEDEF rend visibles les contraintes nationales de l'internationalisation et, plus particulièrement, celles propres à chaque organisation. Plus qu'un modèle français ou italien d'investissement des enjeux européens, il existe des contraintes propres au fonctionnement de chaque organisation au sein de ces espaces nationaux. Or, cette variable est rarement prise en compte dans les travaux analysant les «multi-

41. Entretien avec le permanent du CNPF à Bruxelles entre 1979-1988 et 1996-2003, mai 2010.

42. Entretien un ancien délégué permanent du CNPF à Bruxelles (1988-1996), mars 2012. 
level players » qui se focalisent souvent essentiellement sur les ressources dont dispose l'organisation et rarement sur son fonctionnement. La présence à Bruxelles tient les délégués permanents éloignés des jeux d'organisation et la diffusion de la prise en charge de l'Europe au sein du CNPF-MEDEF aux différents experts techniques concernés ne leur permet pas de faire de leur présence à Bruxelles une ressource valorisable dans l'organisation nationale. Si plusieurs travaux ont documenté les mobilisations des ressources européennes dans des compétitions nationales ${ }^{43}$, nous ajoutons que la capacité des acteurs à bénéficier d'une action multi-positionnée dépend en partie de leur position dans l'organisation.

Malgré son activité circonscrite, le peu de moyens qui lui sont alloués et la difficulté de ses délégués permanents à trouver leur place à l'échelle nationale ou européenne, le Bureau de représentation de la confédération patronale à Bruxelles est conservé, à la différence de la commission Europe du CNPFMEDEF dont l'activité est régulièrement mise en veille. Interroger les logiques de ce maintien revient à préciser le fonctionnement de la confédération patronale française et les tensions entre actions de lobbying à l'échelle européenne et justification de son activité auprès des membres nationaux.

\section{Une présence européenne essentiellement destinée à l'espace national}

Le CNPF-MEDEF est inscrit dans un cadre national, d'où la confédération tire sa reconnaissance juridique et au sein duquel évoluent principalement ses membres. Cette situation particulière a des effets sur la manière dont les sujets européens sont approchés au sein de l'organisation. Comme le rappelle le directeur des affaires européennes du MEDEF :

«Il ne faut pas oublier que nous sommes une organisation nationale avec des membres nationaux. Le retour sur investissement du Bureau de Bruxelles n'est pas du tout perçu par les membres. C'est difficile à évaluer sur le temps court. L'intérêt immédiat n'est pas palpable pour une structure comme la nôtre si on reste dans le lobbying pur ${ }^{44}$ ».

Ainsi, l'orientation des activités du Bureau de Bruxelles vers le seul lobbying n'aurait pas un « intérêt palpable » pour le MEDEF dans les relations avec ses membres. Ce faisant, le directeur des affaires européennes ne dit pas que la confédération patronale ne mène pas d'action de lobbying. Il estime en revanche que la prise en compte des attentes des membres de la confédération rend nécessaire le développement d'autres activités. Il compare ainsi l'activité

43. Roger A., "Jeux d'échelles dans la construction de la représentativité », Gouvernement et action publique, 2012, vol. 2, $\mathrm{n}^{\circ} 2$, pp. 141-166.

44. Entretien avec l'actuel directeur des affaires européennes du MEDEF, juillet 2017. 
du Bureau du MEDEF à Bruxelles à celui de l'Association française des entreprises privées (AFEP), regroupant des très grandes entreprises privées françaises de dimension mondiale, mais sans prétendre à aucune représentativité :

«L'AFEP à Bruxelles ne fait que du lobbying. Nous, dans notre Bureau à Bruxelles, on fait un peu de lobbying, et encore, de moins en moins, mais surtout de l'accueil et du cocooning de nos fédérations qui ont besoin d'informations ou qu'on leur organise par exemple une journée de rencontres sur les additifs alimentaires. On a aussi de l'organisation de visites et de présentation des institutions européennes pour les Medef territoriaux ${ }^{45}$ ».

Si le MEDEF n'est pas une organisation aussi représentative qu'elle le prétend ${ }^{46}$, cette dimension oriente en partie l'activité de ses représentants et salariés. Le MEDEF n'est ni un euro-groupe, ni un groupe d'entreprises, comme l'AFEP. Ses dirigeants affirment s'exprimer au nom des «patrons français». Dans ces conditions, le Bureau de Bruxelles vient justifier l'apport de la confédération patronale à ses membres, en étant davantage une porte d'entrée vers les institutions européennes ou un espace de conseils qu'un moyen d'accumuler une expertise technique particulière. Le Bureau à Bruxelles remplit en partie un rôle de guichet pour les affaires européennes à destination des membres et permet de rendre visible une présence européenne.

La visibilité d'une présence européenne est d'autant plus importante que la prise en charge des sujets européens par les milieux économiques français fait l'objet de nombreuses critiques. Plusieurs rapports, produits par la Chambre de commerce et d'industrie de Paris (CCIP) ou par la Représentation permanente de la France auprès de l'UE ${ }^{47}$, font état de moyens limités développés à Bruxelles, ce qu'ils associent à un « retard français » sur les sujets européens. Ce « retard français » sert d'appui à des critiques récurrentes sur le peu d'investissement des dirigeants de la confédération patronale française à Bruxelles. Le « retard français » participe de cette injonction à être à Bruxelles. Loin d'être un élément descriptif, il est prescriptif et s'apparente à une « rhétorique de l'insuffisance et du changement », dont les premières mobilisations ont été identifiées en France dès le XVIII siècle 48.

\section{Ibid.}

46. Offerlé M., Les Patrons des patrons. Histoire du Medef, Paris, Odile Jacob, 2013, p. 2.

47. «Renforcer le lobbying des entreprises françaises à Bruxelles », Rapport adopté lors de la l'Assemblée générale de la Chambre de commerce et d'industrie de Paris, 2002 ; Marina Féat, L'influence française dans les think tanks traitant des questions européennes, Étude réalisée pour la Représentation permanente de la France auprès de l'Union européenne, juillet 2004 ; "Le Lobbying des entreprises françaises à Bruxelles. Quels progrès depuis 2002 ? ", Rapport adopté lors de la l'Assemblée générale de la Chambre de commerce et d'industrie de Paris, 2005 ; « Lobbying des entreprises françaises à Bruxelles. Nouveau contexte et nouvelles pratiques », Rapport adopté lors de la l'Assemblée générale de la Chambre de commerce et d'industrie de Paris, 2011. 
Si l'idée d'un retard français sur les sujets européens a été infirmée ${ }^{49}$, il demeure régulièrement mobilisé dans les concurrences entre différentes organisations patronales françaises. Maintenir une représentation à Bruxelles, même limitée, rend visible la prise en compte des sujets européens.

La mobilisation de la thématique du retard français n'est pas l'apanage des représentants d'intérêts économiques. Les moyens restreints du Bureau de représentation de la confédération patronale française ont fait l'objet de critiques portées par le pouvoir politique français. Ainsi, en 1989, Édith Cresson, alors ministre des Affaires européennes, écrit à François Perigot, le président du CNPF, pour pointer le retard français sur le lobbying européen :

« Le "lobbying" est avant tout un instrument de communication avec le pouvoir politique que les responsables économiques doivent apprendre à utiliser désormais au niveau européen (...). Nos voisins semblent l'avoir compris plus vite que nous, parfois à nos dépens 50 ».

La ministre des Affaires européennes accompagne sa lettre de plusieurs articles, dont une étude mentionnant les activités du BDI à Bruxelles. Le président du CNPF est alors contraint de rédiger une réponse justifiant de l'action déjà menée et affirmant sa volonté de réinvestir ces questions. Il existe une injonction à être présent à Bruxelles, portée tant par des membres, que par des responsables politiques. Ce « retard français» est établi par rapport à une norme qu'il substantifie. En l'occurrence, le CNPF-MEDEF serait en retard sur les sujets européens parce que son Bureau de représentation est structuré différemment de celui d'autres confédérations nationales. L'imposition progressive de l'idée qu' « être européen signifie avoir des locaux à Bruxelles et un personnel, sinon permanent, du moins dédié à cette activité ainsi localisée 51 ", s'accompagne également d'une injonction de plus en plus forte pour les dirigeants des organisations.

Le Bureau bruxellois participe d'une stratégie d'affichage de l'activité européenne de la confédération patronale, même si elle est portée par d'autres acteurs et définie dans d'autres espaces. De la même manière que les représentants de régions à Bruxelles, en imposant «l'existence de leur profession » auprès des institutions européennes, participent de la légitimation des entités

48. Bouchard J., Comment le retard vient aux Français : analyse d'un discours sur la recherche, l'innovation et la compétitivité, 1940-1970, Villeneuve d'Ascq, Presses universitaires du Septentrion, 2008.

49. Michel H. (dir.), Représenter le patronat européen: Formes d'organisation patronale et modes d'action européenne, Bruxelles, Peter Lang, 2013.

50. Archive nationale (AN), Centre des archives du monde du travail (CAMT), 2000021 79, CNPF, lettre d'Édith Cresson à François Perigot sur le lobbying à Bruxelles, 27 juin 1989.

51. Michel H. et S. Laurens, « Socio-histoire d'un espace de représentation européenne des intérêts patronaux (1960-2004)», op. cit., p. 44. 
régionales comme des acteurs du gouvernement de l'UE ${ }^{52}$, le maintien des délégués permanents légitime le rôle européen du CNPF-MEDEF à l'échelle française.

L'activité des délégués permanents de la confédération patronale française à Bruxelles a été régulièrement redéfinie depuis 1973. Certains se décrivent comme des interlocuteurs des groupes européens et principalement de l'UNICE-BusinessEurope. D'autres insistent sur leur action de soutien à l'action européenne des fédérations nationales membres du CNPF-MEDEF. Tous signalent leur peu de moyens humains et financiers en comparaison de leurs homologues des autres confédérations patronales nationales représentées à Bruxelles. Cette position donne à voir plusieurs éléments contraignant le développement d'une position transnationale. Tout d'abord, ces délégués permanents évoluent dans un espace transnational concurrentiel où ils sont confrontés à d'autres formes de prise en charge de l'européen à Bruxelles, comme l'UNICE. Ensuite, leur position est définie au sein d'un cadre institutionnel, celui de la confédération patronale, où l'expertise européenne est déjà revendiquée par d'autres acteurs disposant d'un profil plus technique. Enfin, leur activité est en partie structurée par la nécessité de se positionner dans l'espace national, que ce soit vis-à-vis de demandes de membres du gouvernement ou d'autres organisations patronales.

L'article documente ainsi les marges de l'Eurocratie à partir d'acteurs disposant de peu de ressources européennes et ne parvenant pas à poursuivre leur carrière dans d'autres organisations de l'espace politique européen. Le maintien de ces acteurs dans cet espace transnational dépend davantage de dynamiques nationales qu'européennes. Malgré cette misère de position, ces acteurs participent à la structuration de l'espace politique européen, notamment en diffusant l'information et en accompagnant les prises de contact. L'attention portée aux contraintes de l'action transnationale éclaire ainsi la structuration des espaces transnationaux par leurs marges.

52. Costa O., "Les représentants des entités infra-étatiques auprès de l'Union. Processus de professionnalisation diversifiés et intérêts communs », op. cit. 